\title{
Des voix de la séduction : à l'écoute de Macbeth
}

\author{
Jean-Louis Claret
}

\section{(2) OpenEdition \\ Journals}

Édition électronique

URL : http://journals.openedition.org/shakespeare/347

DOI : 10.4000/shakespeare.347

ISSN : 2271-6424

Éditeur

Société Française Shakespeare

Édition imprimée

Date de publication : 1 novembre 1999

Pagination : 75-88

ISBN : 2-84269-331-0

Référence électronique

Jean-Louis Claret, "Des voix de la séduction : à l'écoute de Macbeth", Actes des congrès de la Société française Shakespeare [En ligne], 17| 1999, mis en ligne le 01 novembre 2007, consulté le 21 avril 2019. URL : http://journals.openedition.org/shakespeare/347 ; DOI : 10.4000/shakespeare.347 


\section{S H A K E S P E A R E \\ \& $\quad$ L $A \quad$ V O I X}

Société Française Shakespeare

Actes du Congrès de 1999

米米

Textes réunis et présentés par

Patricia DORVAL

publiés sous la direction de

Jean-Marie MAGUIN 


\section{DES VOIX DE LA S ÉDUCTION : A L'ÉCOUTE DE MACBETH}

Il est difficile pour quiconque n'a jamais personnellement eu l'occasion ou le courage de dire les textes de Shakespeare sur une scène de parler pertinemment de la voix, cet aboutissement de l'écriture dramatique. En tant que modeste lecteur je m'efforce en tout cas d'entendre les mots du dramaturge, de jouer mentalement ces drames qui ne prennent vie véritablement que lorsque les acteurs en permettent une incarnation. Le travail que je me propose de présenter ici est donc en partie fondé sur ces représentations. Il est aussi fondé sur des souvenirs de spectacles auxquels j'ai assisté mais le regard que cette étude m'a poussé à porter sur ma propre perception des pièces m'a semblé en soi digne d'intérêt.

$S$ 'interroger sur la signification et la fonction de la voix oblige en effet à mettre le texte en perspective, à replacer au premier plan de l'analyse ce qui échappe au texte et qui pourtant le constitue. C'est au phénomène de séduction opéré par l'entremise de la voix que j'ai consacré la suite de ce travail. Afin d'en éclairer certains mécanismes, je me suis efforcé de déterminer la place de la voix dans le processus qui débouche sur la genèse du texte dramatique. Mais le théâtre est en soi un instrument de séduction qui met parfois en scène la séduction. Une tragédie comme Macbeth, par exemple, présente des personnages tout à la fois repoussants et attachants qui séduisent et détruisent tour à tour. Les jeux de la mort et de l'amour, vers lesquels la séduction conduit, semblent se nicher dans ces instants où les voix se taisent et cèdent la place à un silence éloquent. 


\section{La voix absente}

La voix c'est bien sûr ce souffle qui passe en nous et porte à l'oreille de l'autre les sons qu'on désire y déposer. Sauf exception notable ${ }^{1}$, elle est «cette matérialité du corps surgie du gosier» ${ }^{2}$. Elle est jaillissement, projection de soi et «contagion» de l'esprit récepteur. Elle est le support le plus courant et le plus riche de l'expression en ce qu'elle dit, ce qu'elle laisse entendre et ce qu'elle trahit. "Toute voix se pénètre de ce qu'elle dit» ${ }^{3}$ affirme Roland Barthes mais elle peut aussi contredire le message qu'elle est censée véhiculer: elle semble bénéficier d'une certaine autonomie. Comme le dit Montaigne, «la langue se trahit et la voix se fige à son heure» ${ }^{4}$. La triste histoire d'Actéon que ses chiens ont dévoré parce qu'ils n'avaient pas reconnu ses cris ${ }^{5}$, semble attester la nécessaire adéquation entre la voix et la personne dont elle est l'empreinte. Mais au théâtre, elle demeure avant tout le support du langage, de ces mots fixés sur le papier par l'auteur et qu'elle métamorphose en sons. Une scène dans une pièce de théâtre est constituée de mots en mouvement. Il ne s'agit pas simplement d'associer des voix à des faits que les personnages commenteraient. Le texte dramatique n'est pas un phylactère associé à un tableau vivant. Il est une source dynamique, porteur d'un souffle et l'une des difficultés de ce type d'écriture réside dans le fait que les échanges entre les personnages sont tout d'abord pensés en termes d'écriture. Pierre Larthomas met le doigt sur cette contradiction fondamentale : «Choisir un style, dit-il, c'est [...] définir la nature du compromis entre les deux langages [écrit et oral]. Entreprise difficile, essentiellement en ceci : l'écriture précédant nécessairement le dit, la complexité du langage dramatique tient au fait qu'il est un compromis entre deux langages dont certains caractères sont, dans une grande mesure, opposés. Écrire un bon langage dramatique, c'est unir les contraires» $^{6}$. La voix de l'acteur est le point de rencontre de ces deux constituants.

Tel le poète délaissé par sa muse et qui décrit avec une ferveur désespérée la page blanche qu'il essaie ainsi de remplir, je me suis efforcé de tirer partie de la difficulté que $j$ 'avais à cerner très précisément cette voix théâtrale tellement présente et évanescente lorsqu'il est question d'étudier ou de présenter une œuvre dramatique. Mon malaise était en partie dû au fait que la voix absente et imaginée par le lecteur est bien évidemment différente de celle qui frappe les sens. La présence de la voix est affirmation de la présence de l'Autre qui la perçoit. Parler seul c'est, en ce sens, faire de soi-même l'autre auquel on s'adresse. La perception 
intérieure de la voix de l'acteur (qui correspond aussi à une espèce de dialogue avec soi-même) doit donc être doublée de la perception imaginaire de la perception de la voix de l'acteur. En d'autres termes, le critique doit recréer la voix et la perception qu'il en aurait si celle-ci lui était perceptible. Il devient cet Autre «sensible» et immanent.

La tâche est rendue d'autant plus difficile que si la voix porte le texte, la perception effective de ce dernier tend à la faire disparaitre. Le spectateur écoute les mots du dramaturge et n'entend plus la voix. Elle rappelle cet «objet sans objet» qu'est le miroir. Il est en effet impossible de voir un miroir puisque celui-ci est indissociable du reflet qu'il porte en lui et qui l'anime (au sens étymologique du terme [anima: l'âme, le souffle]). Le reflet cache donc l'objet tout en le révélant; c'est un masque ${ }^{7}$ et en tant que tel il est proclamation de la dissimulation. Ainsi la voix naît-elle et meurt-elle avec le texte qu'elle «incarne». Sa fonction semble prendre le pas sur son être qui ne peut être en partie perçu que lorsqu'il s'agit du murmure ou du cri. Mais ces derniers occupent eux aussi une fonction dramatique qui les place au cœur de la représentation. Toutefois, lorsque la voix devient chant ou dans une certaine mesure poésie (un «cri habillé» disait Max Jacob), le processus s'inverse en ce sens que le timbre de la voix l'emporte parfois plus aisément sur ce qu'elle dit. Dans ses Confessions (Chapitre XXXIII), Saint Augustin fait allusion au "piège» que représente pour lui le chant. Il avoue s'être longtemps laissé séduire par les mélodies et avoir négligé le texte qu'elles devaient accompagner. "Quand il m'arrive d'être plus ému du chant que des paroles chantées, j'avoue que mon péché mérite pénitence, et alors je préférerais ne pas entendre de chants». Bien qu'il «flotte entre le danger du plaisir et la constatation des bons effets» qu'opère la tradition du chant à l'église, Saint Augustin se dit tout de même favorable au maintien de cette institution.

De même que l'attention que l'on porte au propos, le regard peut contribuer à altérer la perception de la voix. Il est intéressant de remarquer que le fait de fermer les yeux donne à l'ouïe une plus grande acuité. Hermia nous donne une leçon à ce sujet puisqu'elle explique que «Dark night, that from the eye his function takes, / The ear more quick of apprehension makes ; / Wherein it doth impair the seeing sense, / It pays the hearing double recompense» ( $A$ Midsummer Night's Dream, III.2.177-80). La voix a un effet métonymique très puissant: «La voix par laquelle, dit Roland Barthes, on reconnaît les autres (comme l'écriture sur une 
enveloppe), nous indique leur manière d'être, leur joie et leur souffrance, leur état ; elle véhicule une image de leur corps et, audelà, toute une psychologie» ${ }^{8}$. Il semblerait que l'ouie et la vue ne puissent additionner leurs acquis respectifs. Les jeux de la bouche et des yeux sont assez fascinants en ce sens qu'ils semblent mener une lutte permanente. L'image du baiser peut permettre d'en appréhender en partie la richesse et les oppositions : on peut en effet «embrasser (un paysage) du regard», mais lorsqu'on embrasse on ferme les yeux (mais il est vrai qu'on parle peu en de telles circonstances...). Je n'irai tout de même pas jusqu'à recommander aux spectateurs de fermer les yeux pendant le spectacle afin d'augmenter leur capacité de perception, mais pourquoi ne pas proposer comme c'est le cas dans Hamlet une pantomime avant la représentation proprement dite afin que cette dernière puisse faire l'objet d'une écoute d'une plus grande richesse? Le Meurtre de Gonzague «sans le son» demeure récréatif; mais à partir du moment où les voix retentissent il devient le piège à souris qu'Hamlet a tendu à son beau-père.

Évoquer les problèmes liés à la voix au théâtre revient à envisager les techniques oratoires mises en œuvre par les acteurs pour engourdir la créature aux cent yeux que les spectateurs sont devenus. Endormir la vigilance d'Argus requiert en effet l'utilisation de la voix jouée et de la voix parlée, la conjonction du texte et de sa mélodie propre. Les acteurs deviennent autant d'instruments autophones, à l'instar d'Hamlet dont les espions du roi prétendent jouer comme ils l'auraient fait d'une flûte (III.2) ${ }^{9}$.

\section{La voix kinesthésique}

La voix est l'un des éléments constitutifs majeurs de l'univers dramatique qui en comporte une multitude ${ }^{10}$. Associée aux gestes elle permet en effet de donner substance aux mots du dramaturge. Mais il ne s'agit pas exactement d'une association, aussi étroite qu'elle puisse être, de deux modes d'expression, à savoir d'un côté le mouvement («le langage des besoins» nous dit Rousseau dans son Essai sur l'origine des langues) et de l'autre la voix (que le philosophe considère comme «le langage des passions»). Ces deux aspects sont en fait inséparables : seule leur imbrication, voire leur fusion, peut permettre à la magie du langage et au charme (dans les différentes acceptions du terme ${ }^{11}$ ) d'opérer. J'en veux pour preuve cette petite histoire chère à Robert Krauss, Professeur de Psychologie à l'Université de Columbia et spécialiste des rapports entre parole et geste. Il s'agit de deux amis qui marchent dans la rue 
par une journée d'hiver particulièrement froide. L'un d'entre eux parle sans arrêt. Quant à l'autre il ne dit pás un mot et garde ses mains profondément enfouies dans ses poches. Il se contente de manifester de temps en temps son approbation par un signe de la tête. Son ami plus loquace finit par lui demander pourquoi il ne parle pas. La réponse est : «Parce que j'ai oublié mes gants». Au delà de l'anecdote il faut voir là le signe de l'interdépendance de la parole et des gestes. Ces derniers ne seraient pas, comme on a coutume de le penser, un langage visuel véhiculant du sens, mais la dimension kinesthésique de la voix. En fait, le mouvement est, d'après Robert Krauss, l'élément indispensable à la recherche de mots dans la banque de données de notre cerveau. Le geste donnerait accès au lexique et ainsi permettrait le jaillissement de la voix. Transposé sur une scène de théâtre, ce phénomène atteste, par la corrélation entre voix et geste, la texture rythmique de la langue dans laquelle le texte est rédigé (on peut imaginer que les acteurs italiens, par exemple, proposent une version très gestuelle des pièces traduites dans leur langue) ${ }^{12}$. Même si ces derniers respectent les consignes que Shakespeare a insérées dans Hamlet et disent leurs textes «trippingly on the tongue» (III.2.2) (à noter que $c$ 'est un moment important en ce sens que le dramaturge fait entendre sa voix), il semble inévitable qu'ils «suit the action to the word, the word to the action» en fonction des impératifs de leur langue maternelle. On peut pousser la réflexion plus avant et se demander si un acteur anglais bouge différemment d'un acteur français, par exemple. De plus, un acteur élisabéthain parlait-il avec ses mains de la manière dont le fait un acteur britannique contemporain? Les gestes ont une voix dont le timbre a certainement évolué notablement... En d'autres termes, on peut se demander s'il n'y a pas une dimension du jeu théâtral qui échappe aux considérations purement dramatiques.

Cette remarque liminaire a pour finalité de démontrer qu'il est difficile d'envisager la voix en dehors du geste qui la constitue. La conjonction et l'accord entre ces deux aspects rend possible la mise en place d'un spectacle qui «fonctionne», en ce sens que les spectateurs sont à même d'y lire, d'y «reconnaître» la projection de leur moi collectif (de leur voix collective ?) et de ce fait à même de succomber à une forme de séduction. La séduction n'est-elle pas, en effet, toujours séduction de soi par l'intermédiaire de l'autre? 


\section{Macbeth et la figure d'Orphée}

La confrontation avec le reflet intègre de la représentation est un espace de séduction dont le vecteur est au premier chef la voix des acteurs. Les spectateurs affirment leur désir d'être séduits du fait même de leur décision de céder à leur pulsion scopique. Se rendre au spectacle c'est en effet vouloir être conquis, vouloir être «mis à part» du monde tangible comme l'indique l'étymologie du verbe séduire (seducere). La dimension orphique du théâtre est donc une donnée fondamentale de cet art et la métaphore du miroir associée à la voix en a déjà annoncé l'immanence. Les jeux de la séduction sont rendus d'autant plus manifestes que des personnages de Shakespeare semblent avoir une certaine conscience de l'effet que produisent leurs paroles sur les autres personnages. Les jeux de l'amour, de la voix et de la séduction dans Macbeth semblent rattacher le héros tragique à la figure mythologique d'Orphée qui eut assez de confiance en son art pour oser, armé de sa seule lyre et de sa voix mélodieuse, aller rechercher Eurydice dans le royaume des morts.

Le parcours de Macbeth peut être considéré lui aussi comme une forme de descente aux Enfers, un enlisement dans le Mal qui a pour corrélât une élévation d'ordre esthétique. Le personnage est investi d'une sensibilité douloureuse alors même qu'il se dépouille de son intégrité morale. Ce phénomène s'accompagne d'une capacité de séduction et d'une force de conviction irrésistibles. Les meurtriers par exemple (III.1) se laissent convaincre et paraissent devant leur seigneur comme des serpents devant leur charmeur. Ils semblent se laisser hypnotiser par sa voix. Macbeth est alors semblable aux sirènes de l'Odyssée qu'évoque Paul-Laurent Assoun et qui «médusent par la voix» ${ }^{13}$. L'hypnose, nous dit d'ailleurs Freud, est fondée sur le pouvoir de la voix dont le regard se fait le complice. «Le regard est la superstition de la parole» ${ }^{14}$ dit encore Paul-Laurent Assoun. Seule la combinaison de ces deux éléments rend l'expérience opérante. Lady Macbeth semble elle aussi être prise dans les rets du chant séducteur puisque son époux, à la suite de sa longue évocation du ballet nocturne des insectes (III.2), constate qu'elle est subjuguée par ses propos: «Thou marvell'st at my words» (54) lui dit-il. Macbeth charme par sa voix et ses mots, comme il fut charmé par ceux des sorcières. «My noble partner / You greet with present grace, and great prediction / Of noble having and of royal hope, / That he seems rapt withal » (I.3.54-7 \& I.5.6). Lors de cette première rencontre les Weird Sisters adoptent une attitude qui relie le geste et la voix puisque «each at once her 
choppy finger laying / Upon her skinny lips» (44-5). Ce rejet de la voix inquisitrice par l'entremise du doigt crochu qui croise et ferme la bouche est une tentative d'abolir l'espace de l'échange et la possible contagion d'un monde par l'autre. La croix ainsi obtenue sera répercutée par l'image hallucinatoire et inversée du «dagger of the mind» que le héros tragique trouvera sur sa route (II.1) ${ }^{15}$. Au rejet direct de la voix correspond alors celui de la vue qui va à son tour entraîner celui de la voix puisque Macbeth s'adressant alors à la terre déclare «Thou sure and firm-set earth, / Hear not my steps, which way they walk, for fear / The very stones prate of my whereabout...». Les sorcières prétendent donc ne pas être des créatures que l'on peut questionner. Leur voix triple va infecter l'esprit de Macbeth qui sera tout de même capable d'entendre celle de sa conscience, ce daïmon qui va, dans un premier temps, le pousser à rejeter la tentation du meurtre ${ }^{16}$. Seule celle de sa femme saura mettre un terme à cette polyphonie inhibante. Contrairement à ce qui se passe dans le récit mythologique selon lequel Orphée sauve les argonautes en couvrant la voix des sirènes grâce au son mélodieux de sa lyre, le combat que se livrent les forces destructrices incarnées ici par les sorcières (au nombre desquelles j'inclus Lady Macbeth) et la vertu (la voix intérieure qui pousse le héros à abandonner tout projet de meurtre) débouche sur la victoire éclatante des forces du mal. Une fois séduit, Macbeth peut séduire tout en se complaisant à écouter la voix off de sa conscience.

Le héros tragique entend cette voix intérieure mais il ne lui obéit pas. Ses apartés attestent la force de cette influence fondée sur le sens du devoir et le pouvoir de l'amour. Mais l'amour a ses voies I voix que la raison ignore et ces dernières peuvent prendre des orientations opposées, d'où la désorientation du personnage. Par amour pour Duncan il doit être fidèle au code sur lequel repose la société et se conformer aux principes d'honneur qui sous-tendent les relations entre les différents intervenants. C'est ce qu'il affirme lorsqu'il énonce les raisons de ne pas tuer le roi : «As I am his kinsman and his subject, / Strong both against the deed; and then, as his host, / Who should against his murtherer shut the door, / Not bear the knife myself» (I.7.13-6). L'amour qu'il porte pour Duncan pousse Macbeth à reculer devant l'acte qui ne pourrait que réduire à néant les injonctions de la «voix du devoir». Celle-ci risque de céder la place au vacarme insoutenable de la culpabilité dévoilée : «His [Duncan's] virtues / Will plead like angels, trumpet-tongu'd, against / The deep damnation of his taking-off» (I.7.18-20). A la peur qu'inspire le son des trompettes de la révélation va correspondre la 
voix entendue lors du passage à l'acte et qui «cried, 'Sleep no more !' to all the house» (II.2.40). Mais la perspective terrifiante de cette «Annonciation monstrueuse» est contrecarrée par le discours de Lady Macbeth qui parvient à faire taire les scrupules de son époux. Sa rhétorique est avant tout fondée sur l'appel à la masculinité de ce dernier. Ses propos sont ponctués d'allusions à ce que doit être a man. («Then you were a man» (I.7.49); «You would / Be so much more the man» (50-1)). Lors de la découverte du corps ensanglanté du roi, Macbeth va d'ailleurs reprendre à son actif cette voix que lui a insufflée son épouse puisqu'il va affirmer devant les seigneurs assemblés : "Who can be wise, amaz'd, temperate and furious, / Loyal and neutral, in a moment? No man» (II.3.106-7). Lady Macbeth s'est alors reconnue comme destinataire de cette voix qui se fait l'écho de la sienne. Le discours intérieur qui prônait l'amour du souverain a cédé la place, dans l'esprit du héros, à la voix d'un autre discours amoureux : celui qui dépasse le devoir pour donner libre cours à la passion. La suite du discours est en effet une déclaration d'amour pour Duncan (l'idole sacrifiée) doublée d'une révélation de l'amour qu'il porte pour sa femme : «Who could refrain, / That had a heart to love, and in that heart / Courage, to make's love known ?» (II.3.114-6) L'évanouissement de Lady Macbeth prend alors une autre dimension. Le phénomène d'écho manifesté par la récurrence du mot man annonce la fusion des deux complices. Ils semblent parler d'une même voix puisqu'au début de la deuxième scène du troisième acte chacun d'eux évoque de la même manière les conséquences morales du crime. D'abord Lady Macbeth dit en aparté : "Nought's had, all's spent, / Where our desire is got without content : / 'Tis safer to be that which we destroy, / Than by destruction dwell in doubtful joy» (4-7), puis Macbeth affirme : «Better be with the dead, / Whom we, to gain our peace, have sent to peace, / Than on the torture of the mind to lie / In restless ecstasy» (19-22). La correspondance entre «doubtful joy» et «restless ecstasy» est symptomatique de la fusion des personnages qui parlent à l'unisson. Les figures de Narcisse et d'Écho se rejoignent ici. Tout d'abord Macbeth s'est conformé à l'image que lui tendait son épouse et à laquelle elle l'identifiait (s'identifiait ?). C'est un Narcisse qui apprend son image et qui se laisse séduire (détruire ?) par son reflet. Ensuite, il y a emprunt de la voix de l'autre par l'adoption d'éléments du langage. Après s'être fait l'écho des propos mystérieux des sorcières («Fair is foul and foul is fair» (I.1.11) / «So foul and fair a day I have not seen» (I.3.38)) il devient le prolongement écholalique de ceux de sa femme. En 
cela, le personnage et son épouse ne font plus qu'un. L'un des deux doit disparaître ${ }^{17}$. Personnage qui se construit en se laissant imposer ses décisions et en empruntant la voix de l'Autre (il est lui aussi à sa façon un «borrower of the night» (III.1.26)), il devient ce nain qu'évoque Angus, et qui sent flotter autour de lui la robe de géant qu'il a volée (V.2.21-2). Le parcours du héros s'achève sur l'image de l'acteur, celui qui porte la voix d'un autre, et le sentiment d'une vacuité que seuls semblent compenser la douleur de la lucidité et la présence sous-jacente du sentiment amoureux.

\section{La voix de l'amour}

«Tout rapport à la voix est forcément amoureux» ${ }^{18}$ affirme Roland Barthes qui se place ainsi dans une perspective platonicienne puisque comme l'affirme Agathon dans Le Banquet «l'amour est poète, et bon poète». Cependant, dans l'œuvre de Shakespeare, ce sentiment est souvent associé au refus de l'oralisation ou du moins à une grande économie de mots. La voix de l'être aimé est douce («She speaks. O, speak again» dit Roméo, II.1.67-8) et unique ( $\ll$ My ears have yet not drunk a hundred words / Of thy tongue's uttering, yet I know the sound», dit Juliette, II.1.1001) mais elle ne semble plus, au sein de la relation amoureuse, être le support du message qu'elle véhicule. «She speaks, yet she says nothing. What of that? / Her eye discourses ; I will answer it» dit encore Roméo (II.1.54-5). Les mots sont dans le regard et la musique est dans la voix : «How silver-sweet sound lovers' tongues by night, / Like softest music to attending ears !» (II.1.210-1). Les amants ne peuvent pas dire la force de l'amour qui les a envahis et même la «riche musique de la langue de Juliette» ${ }^{19}$ ne peut en rendre compte. Dans la première scène du Roi Lear la sincérité lapidaire des sentiments de Cordélie est opposée à la logorrhée hypocrite de Regan et Goneril. «I cannot heave / My heart into my mouth» ditelle (I.1.90-1) comme si la voix s'opposait par nature à la manifestation d'un amour véritable. Les sentiments que l'on pourrait associer à la voix ne seraient-ils pas un manque à gagner pour le cœur humain? En d'autres termes, qui aime la voix de l'autre aime d'autant moins l'autre. L'inadaptation du langage à «dire»l'amour est d'ailleurs, par une étrange ironie, également évoquée par Goneril puisqu'elle affirme qu'elle aime son père «more than words can wield the matter» (54). «Love and tongue-tied simplicity / In least speak most» dit aussi Thésée dans Le songe. Et il affirme que le silence des acteurs fut bien plus éloquent que leurs propos puisque c'est lui qui a su exprimer le plus clairement la sincérité de leur 
émotion. Je ne dirais donc pas avec Roland Barthes que «la voix est, par rapport au silence, comme l'écriture sur le papier blanc» ${ }^{20}$ car de même que chez Mozart les silences sont encore du Mozart, dans l'œuvre de Shakespeare ils ne sont pas absence de paroles. «L'ouie n'est-elle pas, comme le dit d'ailleurs Aristote dans son traité de l'âme, le sens du son et du silence ?» ${ }^{21}$. Ce dernier est le prolongement de la voix, un mode d'expression qui s'adresse peutêtre plus directement à nos émotions.

Dans de nombreuses pièces, le processus dramatique mis en place débouche sur le renoncement à la parole. C'est ce qui se produit dans Hamlet où le héros s'en remet au silence et cède la parole à Horatio. «The rest is silence» dit-il avant de quitter la scène du monde. La mort envahit le personnage qui se sent dériver vers cet ailleurs d'où l'on ne revient pas, cet ailleurs dont la voix s'est tout de même fait entendre au début de la pièce grâce à l'intrusion du fantôme de son père. Il est des cas aussi où les mots deviennent dérisoires et où les personnages renoncent à s'en servir. Iago présente un exemple de mutisme qui rappelle le don de taciturnité octroyé aux sorcières par Satan: «Demand me nothing, what you know, you know, / From this time forth I never will speak word» (V.2.304-5). Quant à Macduff, il renonce aux mots lui aussi lors de sa rencontre avec Macbeth et affirme : «I have no words ; / My voice is in my sword» (V.7.6-7). Les mots (words) cèdent la place à leur substitut anagrammatique ( $s$ word), ce qui implique que le personnage ne met pas un terme à l'expression : seul le support de cette dernière change. Il est des moments où la voix ne peut plus opérer, où l'action (drama) reprend ses droits et affirme sa primauté sur le langage. Il en va de même du discours amoureux. L'amour est du domaine de l'opsis, de ce que l'on manifeste par son comportement. Il est mesuré par la sincérité de l'acte et la folie de Lear est d'avoir imposé à ses filles de le traduire en mots, de l'avoir fait glisser hors du cœur pour le hisser dans la bouche. Cette erreur est fondée sur une appréciation incorrecte de la force du sentiment amoureux dont les nombreux aspects contradictoires ne peuvent être dits. Tullia d'Aragona, dans ses dialogues philosophiques (1547) avec Varchi, intitulés De l'Infinité d'Amour ${ }^{22}$, démontre que «les amants rient et pleurent tour à tour ; ou plutôt [...] ils pleurent et rient en même temps, ils espèrent et redoutent, brûlent et gèlent, veulent et ne veulent pas pareillement, embrassent tout sans étreindre jamais rien; ils voient sans yeux, n'ont pas d'oreilles mais entendent, sans langues poussent des cris, volent immobiles, vivent en mourant, et enfin disent et font tout ce qu'écrivent d'eux les 
poètes unanimes...». Les amoureux n'ont pas de voix pour dire leur passion, leur mode est celui de la mutilation. Tels Écho dont la voix n'est que le pâle reflet de celle de l'aimé dédaigneux, ils sont possédés par la nécessité de devenir l'autre. «L'amour honnête, dit Tullia, qui est propre aux hommes nobles (je veux dire qui ont l'âme délicate et vertueuse, qu'ils soient pauvres ou riches), n'est pas comme l'autre suscité par le désir, mais par la raison ; il a pour fin principale de se transformer en l'objet aimé, désirant aussi la transformation réciproque, de sorte que les deux objets n'en fassent plus qu'un ou en fassent quatre». Dans son Commentarium in Convivium, Ficin nous explique que l'âme de l'amant meurt en lui et vit dans le corps de l'aimé. Si le sentiment n'est pas réciproque, l'âme meurt car elle n'a plus de «domicile». Dans l'amour réciproque, il y a double mort et double renaissance en l'autre. Les sonnets contiennent de nombreuses références à ce phénomène (Sonnets 22, 24, 31, 42,46) mais Romeo and Juliet en présente une illustration très claire puisque le jeune homme affirme que son âme vit désormais dans le corps de Juliette : «It is my soul, that calls upon my name» (II.2.165). Dans Two Gentlemen of Verona, après avoir été banni de la Cour, Valentine dit : «O thou that dost inhabit in my breast, / Leave not the mansion so long tenantless... / Repair me with thy presence, Silvia» (V.4.7-11).

Seule la présence de l'être aimé peut sauver. Il n'est pas ici question de sa voix. Ce phénomène est peut-être dû au fait que la vie des amants s'étiole dans un mimétisme sans issue car l'amour a «détruit» (séduit?) leur âme. Une fois privée de ce souffle fondamental, la voix n'est plus que dissonance et source de division.

Jean-Louis CLARET Université de Nancy II

\section{NOT E S}

${ }^{1}$ Je pense en particulier à la Pythie qui, à en croire Henry Boguet dans son Discours exécrable des sorciers (Rouen, 1603), "parlait par les parties basses et honteuses». 
${ }^{2}$ Définition proposée par Roland Barthes dans L'Obvie et l'obtus : Essais critiques III, coll. Essais, Paris, Seuil, 1982, p. 226. A noter que la notion de matérialité pousse Roland Barthes à rejeter l'idée de souffle.

${ }^{3}$ Op. cit., p. 247.

${ }^{4}$ Michel de Montaigne, Essais, XXI «De la force de l'imagination».

${ }^{5}$ Il faut tout de même reconnaître que la transformation d'Actéon en cerf n'a pas été propice à sa reconnaissance...

${ }^{6}$ Pierre Larthomas, Le Langage dramatique, Presses Universitaires de France, Armand Colin, 1972, p. 177.

${ }^{7}$ Mais sous ce masque il n'y a pas de visage.

${ }^{8}$ Roland Barthes, L'Obvie et l'obtus, op. cit., p. 225.

9 «Why, look you now, how unworthy a thing you make of me. You would play upon me, you would seem to know my stops, you would pluck out the heart of my mystery, you would sound me from my lowest note to the top of my compass; and there is much music, excellent voice, in this little organ, yet cannot you make it speak. 'Sblood, do you think I am easier to be played on than a pipe ? Call me what instrument you will, though you fret me, you cannot play upon me» (III.2.354-63).

${ }^{10}$ Roland Barthes définit la théâtralité comme «une épaisseur de signes» (Essais critiques, coll. Point Essais, 1964, p. 267, chapitre intitulé «Littérature et signification»).

${ }^{11}$ Charme au sens d'un ensorcellement par la magie du théâtre et charme qui entraîne la séduction.

${ }^{12}$ Certaines personnes, nous signale R. Krauss, font 40 fois plus de gestes que d'autres pour exprimer les mêmes idées...

${ }^{13}$ Paul-Laurent Assoun, Leçons psychanalytiques sur le regard et la voix, vol. 1, Paris, Anthropos, 1995, p. 79. L'auteur évoque en une autre occasion le «filet de voix» des sirènes, ces «voix à plumes», p. 71-2. A noter que dans Le Banquet, Alcibiade dit éviter Socrate et «être forcé de [se] sauver en [se] bouchant les oreilles comme devant les Sirènes, sinon [il] risquerai[t] de vieillir à ses côtés» tellement le pouvoir des paroles de ce dernier est grand. Alcibiade le compare au satyre Marsyas qui charmait les hommes grâce au pouvoir de sa flûte. Discours d'Alcibiade: Éloge de Socrate, 215a-216c.

14 Paul-Laurent Assoun, op. cit., p. 81.

${ }^{15}$ On peut également voir là l'image de la croix inversée qui rattache ces créatures aux éléments caractéristiques des messes noires au cours desquelles les symboles conventionnels étaient inversés.

${ }^{16}$ En -399, Socrate, âgé de soixante-dix ans, définit lui-même ce qu'il entend par le mot daimôn (Platon, Apologie, 31) : «C'est quelque chose qui a commencé dès mon enfance, une certaine voix qui, lorsqu'elle se fait entendre, ne me prescrit jamais de faire, mais me détourne de ce que je m'apprêtais à faire». C'est-à-dire c'est un chuchotement apotropaïque, qui révoque, qui inhibe, qui suspend, qui retient. (Commentaire de Pascal 
Quignard dans la préface du Démon de Socrate d'Apulée, Rivages Poche, p. 21).

${ }^{17}$ Ce phénomène ne va pas sans rappeler celui qui se produit avec le personnage de Fou dans Le Roi Lear.

${ }^{18}$ L'Obvie et l'obtus, p. 247. Il dit encore (à la même page) : «Il n'y a aucune voix humaine au monde qui ne soit objet de désir - ou de répulsion».

${ }_{19}$ "And let rich music's tongue / Unfold the imagined happiness that both / Receive in either by this dear encounter» (II.4.27-9).

${ }^{20}$ Op. cit., p. 225.

${ }^{21}$ De l'âme, II.8.

22 Dialogo della Signora Tullia d'Aragona della Infinità di Amore (1547). Il me semblait naturel d'aller chercher autorité en amour auprès d'une femme. N'est-ce pas ce que Socrate a fait en son temps en allant consulter l'Étrangère de Mantinée : Diotime ? Le Banquet, 201d-212d. 